in anderen Teilen der Mathematik Grenzwerte mit bedingt konvergenter Annäherung zugelassen.

$F$.

\title{
Von Strecke, Quadrat und Würfel zum bestimmten Integral.
} Von G. Hering. IV + 135 S., B. G. Teubner, 1910.

Der Zweck dieser Schrift ist es, die Auswertung einiger praktisch wichtiger Integrale aus der Geometrie und Mechanik, auf elementare Entwicklungen gestützt, für einen weiteren Leserkreis verständlich darzustellen. Der Verfasser beginnt dabei zwar mit den einfachsten Aufgaben der messenden Geometrie, scheint aber doch die ganze Elementarmathematik als einigermaßen bekannt vorauszusetzen. Das Hauptgewicht wird auf die Klarlegung solcher Fragen gelegt, die im Schulunterricht gewöhnlich nicht hinreichend ausführlich dargestellt werden, besonders auf die Erklärung der infinitesimalen Begriffe. Gerade aus diesem Grund verdient die Schrift das Interesse der Lehrer. Der Begriff der Integration wird zunächst an den einfachsten ebenen Flächenstücken, eben- und krummflächigen Körpern erläutert, dann aber auch, stets mit Filfe von Figuren, an den Funktionen $a x \mathrm{n}$, $\sin x, \cos x$ und etwas komplizierteren geometrischen und mechanischen Aufgaben. Jeder infinitesimalen Betrachtung geht die Anleitung zu einer physikalischen Messung voraus; die Unterscheidung der unendlich kleinen Größen verschiedener Ordnung wirả durch numerische Abschätzung und durch überaus sorgfältige Zeichnungen verdeutlicht. - - In den meisten elementaren Büchern über Infinitesimalrechnung bildet die Erklärung and Verwendung des Grenzverfahrens den heiklen Punkt. Der Verfasser dieser Schrift hingegen scheut keine Wiederholung, um darin Klarheit za schaffen. Freilich dürfte gerade durch die Hänfung der Argumente manches weniger deutlich geworden sein. Hat man. z. B. eine krumme Linie zwischen zwei Polygone eingeschlossen, deren Flächeñzahlen bei hinreichend feiner Teilung sich beliebig wenig von einander unterscheiden und dabei eine algebraisch bestimmte Maßzahl eingrenzen, so genügt der einfachste SchluB, um die Gleichheit dieser Mafizahl und des fraglichen Flächeninhaltes festzustellen; verwendet man aber ein - wenn auch physikalisch noch so plausibel begründetes - Argument dafür, daß man die Differenz der Polygone "vernachlässigen" könne, so ist damit jener Schluß nicht klar gemacht, sondern verwischt, was gerade mit dem pädagogischen $Z$ weck, den der Verfasser verfolgt, nicht übereinstimmt. Die Einführung der Analysis in den Unterricht verlangt an diesen kritischen Stellen vollkommene Einfachheit and Bestimmtheit der Darstellung, die in den wenigsten Lehrbüchern zu finden ist. - Von kleinen Versehen wäre noch die Behauptung zu erwähnen, daß man aus der Unendlichkeit der Reiho für $\pi$ anf die Irrationalität dieser Zahl schließen könne (Seite 18), ferner die Angabe (Seite 60), daß das Volumen des schiefen Prismas nur durch infinitesimale Teilung auszumessen sei. Nichtsdestoweniger ist das Buch eine didaktisoh interessante und beachtenswerte Erseheinung. $F$.

Annuaire pour l'an 1912, publié par le Bureau des Longitudes, Paris, Gauthier-Villars. Preis Fr. 1.50.

Dieser Jahrgang enthält anßer dem üblichen astronomischen Kalendarium vorwiegend astronomische, physikalische und chemische Angaben, außerdem noch ausführlicheres über veränderliche Sterne. Beigegeben wird eine Abhandlung 